\title{
Correction to: Natural Excipients in Pharmaceutical Formulations
}

Pradeep Singh, Garima Mishra, and Subas Chandra Dinda

\section{Correction to: \\ Chapter 40 in: S. C. Mandal et al. (eds.), Evidence Based Validation of Traditional Medicines, https://doi.org/10.1007/978-981-15-8127-4_40}

The original version of this chapter was inadvertently published with dual affiliations for Dr. Dinda which has been corrected now as follows:

S. C. Dinda

Department of Pharmaceutics, College of Pharmacy, Teerthanker Mahaveer University, Moradabad, UP, India 